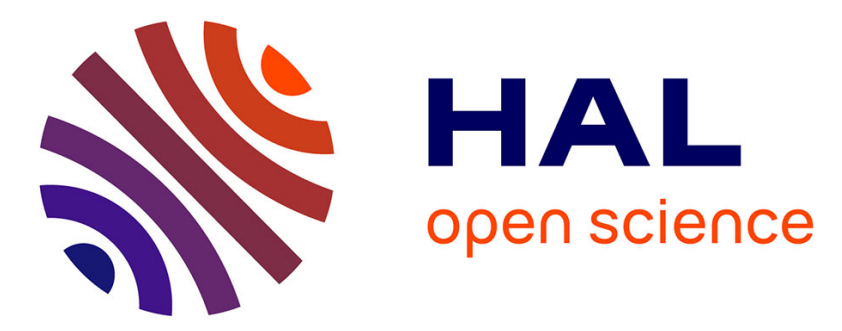

\title{
Gap size effects on centrifugally and rotationally driven instabilities
}

Innocent Mutabazi, Christiane Normand, Eduardo Wesfreid

\section{To cite this version:}

Innocent Mutabazi, Christiane Normand, Eduardo Wesfreid. Gap size effects on centrifugally and rotationally driven instabilities . Physics of Fluids A: Fluid Dynamics (1989-1993) , 1992, 4 (6), pp.1199-1205. 10.1063/1.858238 . hal-01811164

HAL Id: hal-01811164

https://hal-normandie-univ.archives-ouvertes.fr/hal-01811164

Submitted on 8 Jun 2018

HAL is a multi-disciplinary open access archive for the deposit and dissemination of scientific research documents, whether they are published or not. The documents may come from teaching and research institutions in France or abroad, or from public or private research centers.
L'archive ouverte pluridisciplinaire HAL, est destinée au dépôt et à la diffusion de documents scientifiques de niveau recherche, publiés ou non, émanant des établissements d'enseignement et de recherche français ou étrangers, des laboratoires publics ou privés. 


\title{
Gap size effects on centrifugally and rotationally driven instabilities
}

\author{
Innocent Mutabazi ${ }^{j)}$ and Christiane Normand \\ Service de Physique Théorique, Direction des Sciences de la Matière du CEA, Centre d'Etudes de Saclay, \\ F-91191 Gif-sur-Yvette Cedex, France \\ Jose Eduardo Wesfreid \\ Laboratoire de Physique et Mécanique de la Matière Hétérogène, URA CNRS 857, Ecole Supérieure de \\ Physique et de Chimie Industrielles de Paris, 10, rue Vauquelin, F-75231 Paris Cedex 05, France
}

(Received 1 March 1991; accepted 30 December 1991)

\begin{abstract}
The rotation effects on centrifugally driven instabilities in curved channel flow with a finite gap are investigated. An inviscid criterion of stability is formulated to explain the behavior of the flow when rotation and curvature effects compete to either stabilize or destabilize the flow. The stability of curved Poiseuille flow with finite gap size is studied, and it is shown that the asymmetry between the directions of rotation is enhanced when the gap size increases.
\end{abstract}

\section{INTRODUCTION}

The importance of flows with curved streamlines has drawn much attention from scientists since the end of last century because of their applications in everyday life and in technology. A decisive step toward an understanding of instabilities in curved geometries has been done by Taylor ${ }^{1}$ who studied the stability of Couette flow between independently rotating cylinders. Though they have not received comparable interest, open flows in curved channels are progressively well understood. The Poiseuille flow induced by an external pressure gradient in a curved channel formed by two concentric cylinders has been investigated theoretical$1 y^{2-4}$ and experimentally. ${ }^{5-7}$ The combination of the Couette and Poiseuille flows has been achieved in a so-called TaylorDean system and has shown to be rich in patterns, in particular stationary axisymmetric and traveling inclined rolls have been observed ${ }^{8}$ according to theoretical predictions. ${ }^{9}$ All these instabilities are due to the curvature of streamlines inducing a centrifugal force which unbalances the radial pressure gradient. As a result, longitudinal rolls are formed and superimposed upon the base flow.

Another source of longitudinal rolls is the rotation of the flow system around its own axis. Indeed, the plane channel flow, which is stable to spanwise infinitesimal perturbations, becomes unstable under slow rotation about its own axis and longitudinal rolls appear in the flow. ${ }^{10-13}$ Positive rotation (i.e., the rotation vector is parallel to the shear vorticity) of the boundary layer over a plane plate is destabilizing while negative rotation stabilizes the flow. ${ }^{14}$ Therefore, the rotation of the flow system generates a Coriolis force which has a destabilizing or stabilizing effect on the flow depending on its interaction with the shear force. Thus, there is a similarity between streamline curvature and the flow rotation.

The interaction between rotation and streamline curvature may generate new patterns and, in particular, oscillatory modes. In fact, the stability analysis of a curved Poi-

\footnotetext{
a) Present address: Department of Physics, The Ohio State University, 174 West, 18th Avenue, Columbus, Ohio 43210.
}

seuille channel flow rotating around its own axis has led to the following theoretical results: ${ }^{15}$ in the control parameter space there are two branches of stability, one corresponding to stationary modes induced by centrifugal force, the other to stationary modes induced by rotation. These results were confirmed experimentally. ${ }^{15}$ In the neighborhood of the point where the two branches intersect, oscillatory modes were predicted but not observed. The curved channel flow rotating around its own axis exhibits patterns similar to those observed in a Taylor-Dean system. ${ }^{8.9}$

In this paper, we are concerned with isothermal centrifugal instabilitics under the Coriolis force created by rotation of the frame of reference about the axis of flow geometry. In Sec. II, we give the linear stability equations of a rotating curved channel flow with a finite gap. The results of the linear stability analysis are summarized in Sec. III and discussed in Sec. IV. For a better understanding of the mechanism responsible for the rotationally and centrifugally driven instabilities, we reformulate the Rayleigh circulation criterion, including rotation effects. A comparison with the Taylor-Dean system is done explicitly. The rotation effects on Couette flow and on boundary-layer flow over a curved plate are briefly considered using the Rayleigh criterion. A short review of a few hydrodynamic systems which exhibit Hopf bifurcation from the base state is given before our concluding remarks.

\section{LINEAR STABILITY OF ROTATING CURVED CHANNEL FLOW}

We consider a Newtonian incompressible fluid of density $\rho$ and kinematic viscosity $v$ in the curved channel formed by two coaxial cylinders of radius $R$ and $R+d$ (Fig. 1). In a rotating frame of reference, the flow velocity $v$ and pressure $P$ fields are described by the following equations: ${ }^{16}$

$$
\begin{aligned}
& \frac{\partial \mathbf{v}}{\partial t}+(\mathbf{v} \cdot \nabla) \mathbf{v}-2 \mathbf{v} \times \mathbf{\Omega}=-\boldsymbol{\nabla}\left(\frac{P}{\rho}\right)+v \Delta \mathbf{v}, \\
& \operatorname{div} \mathbf{v}=0 ;
\end{aligned}
$$

here $P$ is the sum of the usual pressure term and the centrifu- 


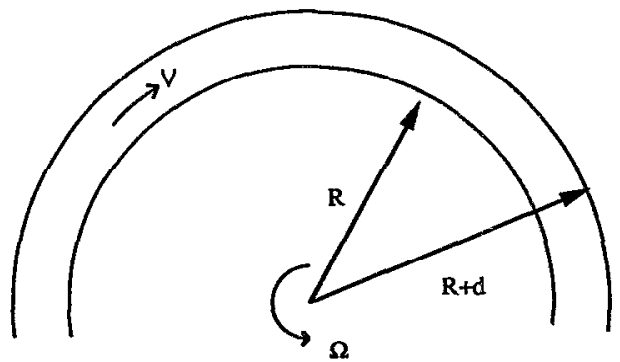

FIG. 1. Sketch of the flow geometry: flow between two fixed coaxial cylindrical surfaces under constant external pressure gradient. The rotation axis coincides with that of the system.

gal energy term $\left(-\frac{1}{2} \Omega^{2} r^{2}\right)$ associated with the rotation of the frame of reference as a whole. Experiments are mostly performed in two cases: (1) the rotation axis coincides with that of the local shear vorticity, in which case, it does not affect the base flow but modifies only the radial pressure gradient; and (2) the rotation axis is orthogonal to that of the hydrodynamical system and the base flow acquires two velocity components. ${ }^{17,18}$

In the following we will consider the rotation of the channel around its own curvature axis, so the base flow solution is unaffected by the rotation and remains two dimensional for low velocities: $\mathrm{V}=[0, V(r), 0]$, the expression for $V(r)$ is given in Ref. 16. The radial variable $r$ can be scaled as follows: $r=R(1+\delta x)$, where $\delta=d / R$ is the curvature parameter or the scaled gap size. In units of the mean velocity $V_{m}=(1 / d) \int_{R}^{R+d} V(r) d r$ across the channel radial section, the azimuthal velocity profile reads

$V(x)=-F^{-1}\left[\frac{\log c}{c}+\frac{(1+\delta)^{2}}{2 \delta+\delta^{2}}\left(\frac{1}{c}-c\right) \log (1+\delta)\right]$,

where

$$
F(\delta)=-\left(\frac{1}{2}+\frac{\delta}{4}-\frac{(1+\delta)^{2} \log ^{2}(1+\delta)}{\delta^{2}(2+\delta)}\right),
$$

with $c=1 /(1+\delta x)$.

In order to study the linear stability of the flow, we superimpose a perturbed flow field $\left(\mathbf{v}^{\prime}, p^{\prime}\right)$ to the base flow field $(V, P)$. The governing equations for the perturbations are obtained by substitution of $\mathbf{v}=\mathbf{V}+\mathbf{v}^{\prime}, p=P+p^{\prime}$ into Eqs. (1) and (2) and neglecling all quadratic terms in the perturbations. If the time, the axial coordinate, and the perturbative pressure are expressed, respectively, in units of $d^{2} / v, d$, and $\rho(v / d)^{2}$, and scaling the azimuthal variable $\theta$ and the velocity as follows,

$$
\theta=\delta^{1 / 2} y, \quad \mathbf{v}^{\prime}=\left(u, \delta^{-1 / 2} v, w\right)(v / d),
$$

we obtain the following nondimensional equations:

$$
\left(L_{0}+c^{2} \delta^{2}\right) u+2 c^{2} \delta \frac{\partial v}{\partial y}-2 \operatorname{De}(c V+\operatorname{Ro}) v=-\frac{\partial p}{\partial x},
$$

$$
\begin{aligned}
& \left(L_{0}+c^{2} \delta^{2}\right) v+2 c^{2} \delta^{2} \frac{\partial u}{\partial y}+\operatorname{De}[D V \\
& \quad+\delta(c V+2 \mathrm{Ro})] u=-c \delta \frac{\partial p}{\partial y}, \\
& L_{0} w=-\frac{\partial p}{\partial z} \\
& \frac{\partial u}{\partial x}+c \delta u+\frac{\partial v}{\partial y}+\frac{\partial w}{\partial z}=0
\end{aligned}
$$

where $D=d / d x$ and the linear operator

$$
L_{0}=\frac{\partial}{\partial t}+c \operatorname{De} V \frac{\partial}{\partial y}-\frac{\partial^{2}}{\partial x^{2}}-\frac{\partial^{2}}{\partial z^{2}}-c \delta \frac{\partial}{\partial y}-c \delta^{2} \frac{\partial^{2}}{\partial y^{2}} .
$$

Thus, the problem is characterized by a triplet of parameters: the dimensionless curvature factor $\delta=d / R$, the Dean number $\mathrm{De}=\left(V_{m} d / v\right) \delta^{1 / 2}$, and the rotation number Ro $=\Omega R / V_{m}$. We have defined the rotation number as the ratio between two characteristic velocities, but it is related to that used in the previous studies, ${ }^{15}$ by $\widetilde{\mathrm{R}} \mathrm{o}=\Omega d / V_{m}=\delta$ Ro. In Eqs. (5) and (6), the rotation terms are added to those accounting for the centrifugal instability. Then, one would expect that the Coriolis force and the centrifugal force act either to enhance or to cancel each other.

In order to study the conditions under which infinitesimal perturbations will develop in the flow, we expand the perturbations in the Fourier-Laplace modes of the form: $\exp [s t+i(q z+p y)]$ where $p$ and $q$ are the azimuthal and axial wave numbers, respectively. The axial wave number $q$ is real because the perturbations must be bounded at infinity in the axial direction. In open flows such as those with a boundary layer, the azimuthal wave number $p$ may be a complex quantity the real part of which describes the spatial growth of the amplitude of perturbations. ${ }^{19}$

The marginal stability state corresponds to those perturbations with $\operatorname{Re}(s)=0$ : the stationary perturbations $(s=0)$ and the oscillatory perturbations $(s=i \omega \neq 0)$. Substitution of the Fourier-Laplace modes in the perturbation equations with homogeneous boundary conditions leads to an eigenvalue problem. This consists in finding a relation between the flow parameters ( $\mathrm{Ro}, \mathrm{De}, \delta$ ) and the perturbation characteristics $(s, q, p)$ of the type

$$
F\left(\mathrm{Ro}, \mathrm{De}, \delta_{;} s, q, p\right)=0 \text {. }
$$

This eigenvalue problem is solved numerically by the method which has been proposed by Rober ${ }^{20}$ and has been successfully used to study the linear stability of many hydrodynamic systems among which are the Taylor-Couette ${ }^{21}$ and Taylor-Dean ${ }^{8}$ problems.

\section{RESULTS}

We have calculated the critical points as functions of the rotation ratio for different values of the gap size $\delta \in[0,1]$. The topology of the neutral stability curve has been given in detail in Ref. 15. Here we will only focus on the variation of instability onset parameters, due to the finite gap size. The resulting phase diagram $\left(\widetilde{\mathrm{R}}, \mathrm{De}_{c}\right)$ contains two stationary 


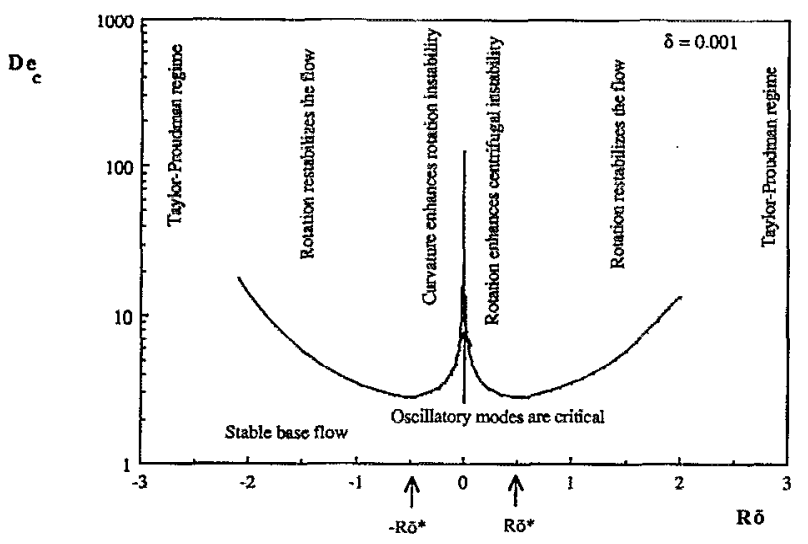

FIG. 2. Phase diagram ( $\tilde{\mathrm{R}}_{\mathrm{o}}, \mathrm{De} \mathrm{e}_{\mathrm{c}}$ ): different regimes shown for $\delta=0.001$.

mode branches that intersect in the vicinity of $\widetilde{\mathrm{R}} 0=-\delta$ (Fig. 2). In that region, critical states are oscillatory ${ }^{15}$ as in the Taylor-Dean problem, ${ }^{8}$ however, the extent of this region in the phase diagram does not vary sensitively with the curvature parameter $\delta$. In the absence of rotation, i.e., for Ro $=0$, we have found that the finite gap size of the curved channel delays the instability threshold in agreement with previous results. ${ }^{22}$

Higher rotation rates $(|\mathrm{Ro}|>1)$ restabilize the flow and the value of Ro* at which restabilization begins depends on the gap size $\delta$. We have observed that, in the case of small gap size $(\delta<0.01)$, the threshold of the instability decreases for $|\widetilde{R} o|<1 / 2$ which expresses a destabilization effect, but for $|\widetilde{R} \mathrm{o}|>1 / 2$, the threshold increases strongly and the flow is restabilized. The quantity $\widetilde{\mathrm{R}} \mathrm{O}^{*}=1 / 2$ is independent of $\delta$ for small gap size. For moderate and large gap size $(\delta \geqslant 0.01)$, the quantity $\widetilde{R} \mathrm{o}^{*}$ becomes sensitive to the gap size $\delta$ [Fig. $3(\mathrm{a})]$ and the correspondent critical parameters $\mathrm{De}_{c}^{*}=\mathrm{De}_{c}\left(\widetilde{\mathrm{R}} o^{*}\right)$ and $q_{c}^{*}=q_{c}\left(\widetilde{\mathrm{R}} o^{*}\right)$ are also dependent upon $\delta$ [Figs. 3(b) and 3(c)].

For highest rotation rates, the Coriolis force restabilizes the flow and leads to the Taylor-Proudman two-dimensional regime as in the straight rotating Poiseuille channel flow. ${ }^{11}$ The asymptotic value of $\widetilde{\mathrm{Ro}}^{+}$at which there is a complete restabilization depends on the curvature parameter $\delta$ : for small gap size $\delta<0.01$, it is given approximately by $\left|\widetilde{R}_{0}{ }^{+}\right|=3$, but for wide gap, the quantity $\left|\widetilde{R}_{0}{ }^{+}\right|$decreases with $\delta$. The Taylor-Proudman regime corresponds to small Rossby numbers (high rotation ratio Ro) and to small Ekman number values (large Dean number) ${ }^{23}$

\section{DISCUSSION}

\section{A. Generalization of Rayleigh's circulation criterion}

In order to better understand the mechanism responsible for the different stability branches of the phase diagram $\mathrm{De}_{c}(\widetilde{\mathrm{R}} \mathrm{o})$ and the origin of the oscillatory modes, we formulate a stability criterion including both Coriolis and centrifugal force effects. This is done by using a displaced particle argument. ${ }^{24}$
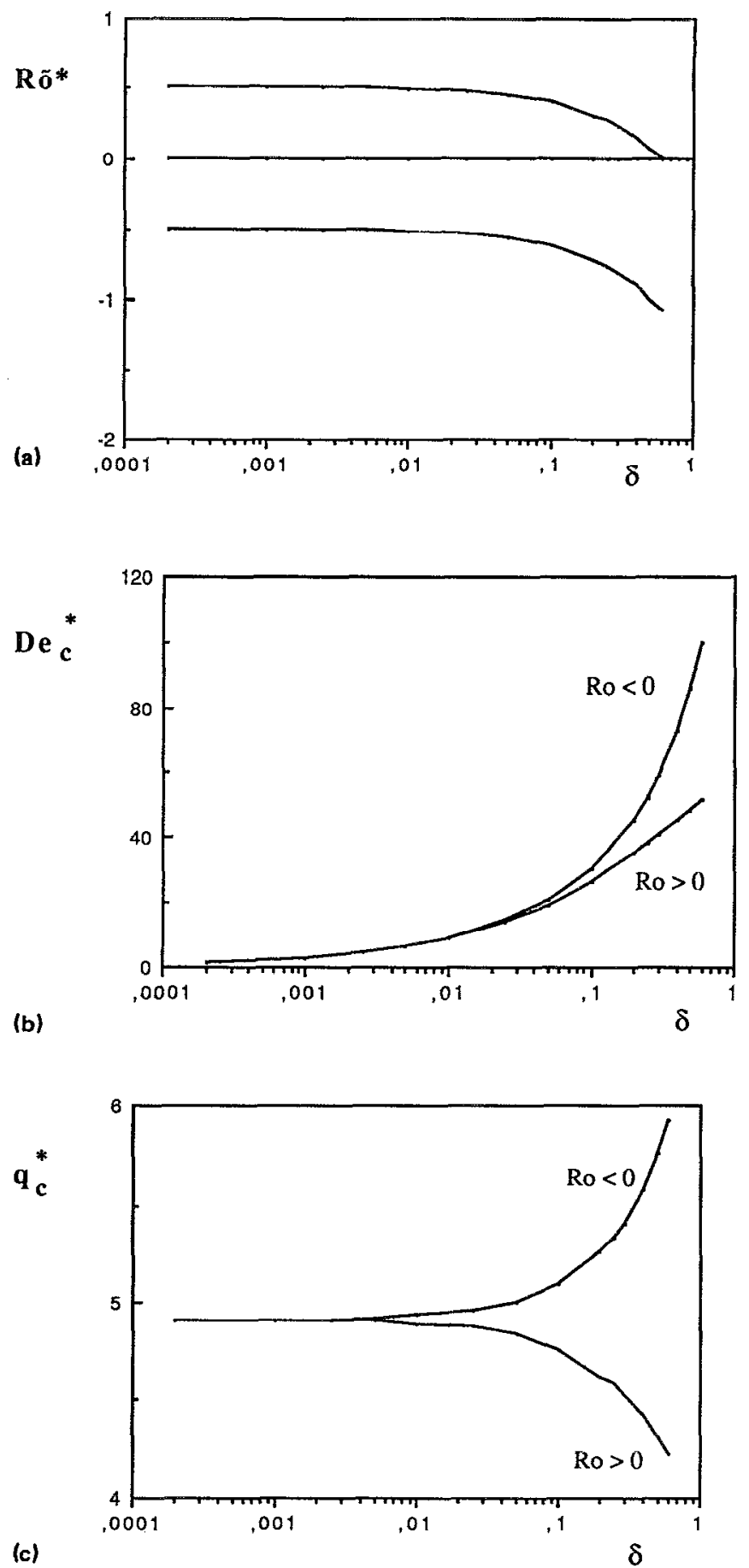

FIG. 3. Wide gap size effects on critical parameters at the restabilization point: (a) $\widetilde{\mathrm{R}}^{*}(\delta)$; (b) $\operatorname{De}_{c}^{* k}(\delta)$; (c) $q_{c}^{*}(\delta)$.

A fluid particle with velocity $V_{1}$ is displaced from its original position $r$ to the new position $r+d r$ so fast that the viscous dissipation does not take place $\left[d r / v_{r} \ll(d r)^{2} / v, v_{r}\right.$ is the displacement speed], then the angular momentum of the displaced particle is conserved and the displaced particle acquires velocity $V^{\prime}$ at the new position (Fig. 4). In the absence of rotation, the velocity $V^{\prime}$ would be given by $V^{\prime}=V_{1} r /(r+d r)$, but we have to add a velocity contribu- 


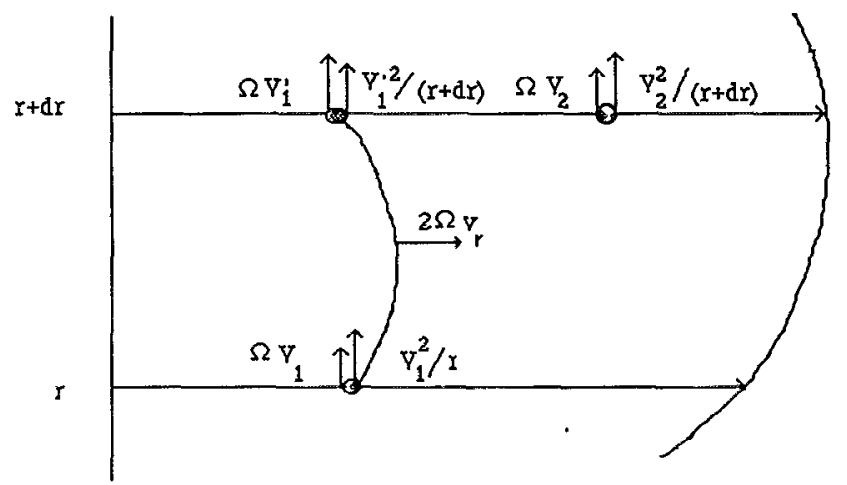

FIG. 4. Sketch for formulation of generalized inviscid Rayleigh discriminant using the displaced particle argument: shown are Coriolis and centrifugal accelerations of the particle at positions $r$ and $r+d r$.

tion from Coriolis acceleration so that, to the first order in $d r, V^{\prime}=V_{1}-\left(2 \Omega+V_{1} / r\right) d r$. The radial pressure gradient acting at the displaced particle at this position $r+d r$ is

$$
\left.\frac{1}{\rho} \frac{\partial P^{\prime}}{\partial r}\right|_{r+d r}=2 \Omega V^{\prime}+\frac{V^{\prime 2}}{(r+d r)} .
$$

This pressure gradient is to be compared with the radial pressure gradient of the nonperturbed flow particle at the same position

$$
\left.\frac{1}{\rho} \frac{\partial P}{\partial r}\right|_{r+d r}=2 \Omega V_{2}+\frac{V_{2}^{2}}{(r+d r)},
$$

where $V_{2}=V(r+d r)=V(r)+(d V / d r) d r$. The restoring force per unit volume acting on the displaced particle is $d F=\left.\left(\partial P / \partial r-\partial P^{\prime} / \partial r\right)\right|_{r+d r}$. After few algebraic calculations, one obtains $d F=\rho \Phi(r) d r+O\left(d r^{2}\right)$, where

$$
\Phi(r)=2 \Omega\left(\frac{d V}{d r}+2 \Omega\right)+6 \frac{\Omega V}{r}+2 \frac{V}{r}\left(\frac{d V}{d r}+\frac{V}{r}\right) .
$$

The quantity $\Phi(r)$ can be called the generalized Rayleigh discriminant. The first two terms give the Rayleigh discriminant for rotating plane channel flow (coupling between rotation and absolute shear vorticity in the inertial frame of reference) as formulated in Ref. 24 . The last two terms give the Rayleigh discriminant for centrifugal instability without rotation and the intermediate term is the coupling between rotation and curvature. The condition for amplifying perturbations driven by either the centrifugal force or Coriolis force is given by $\Phi(r)<0$. The flow is therefore subdivided into layers of different stability depending on the sign of $\Phi(r)$ : it will be unstable in the region where $\Phi(r)<0$ and stable when $\Phi(r)>0$. To have a better insight of this instability mechanism, the discriminant $\Phi(r)$ is written in the form $\Phi(r)=\left(V_{m} / d\right)^{2} \Phi(x)$, where the nondimensional discriminant is given by

$$
\Phi(x)=2 \delta\{(\mathrm{Ro}+c V)[D V+\delta(c V+2 \mathrm{Ro})]\} .
$$

Of interest are the following special cases: the small gap approximation limit $(\delta \rightarrow 0)$ for low and high rotation regimes, and wide gap for high rotation regime.

\section{B. Small gap approximation and the low rotation regime}

In the small gap approximation for moderate values of the rotation number Ro $O(1)$, the discriminant becomes

$$
\Phi(x)=2 \delta\left(\text { Ro }+V_{0}\right) D V_{0},
$$

where $V_{0}=6 x(1-x)$ is the base flow velocity profile in the small gap approximation. The above discriminant should be considered the Rayleigh discriminant for the equivalent velocity profile $V_{e}=V_{0}+$ Ro. The condition $\Phi(x)=0$ gives the frontiers between potentially unstable and potentially stable layers of the fluid in the channel width [Fig. 5(a)]. For Ro $\geqslant 0$, the flow consists of two layers, with the one close to the outer cylindrical wall $(x=1)$ centrifugally unstable
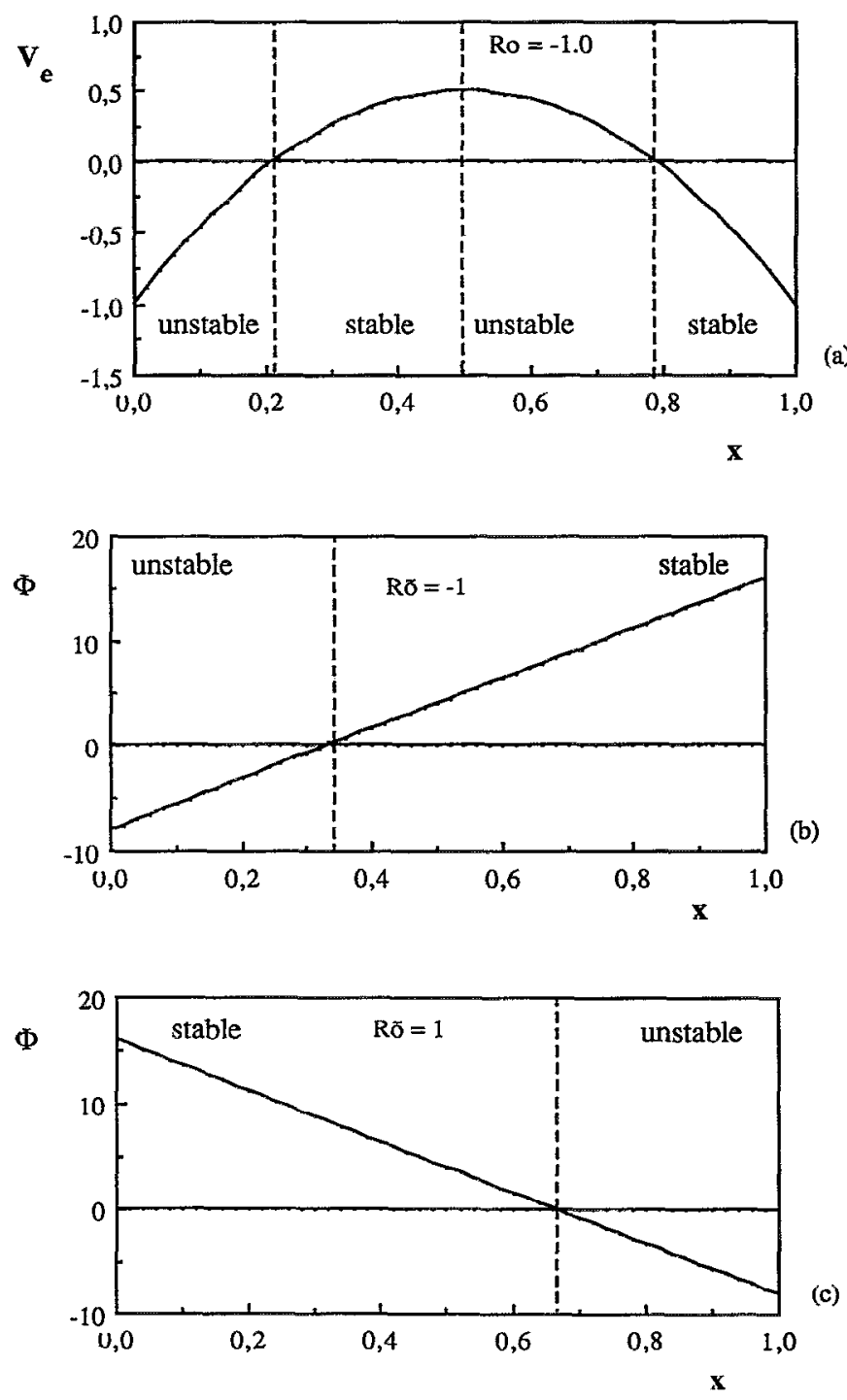

(c)

FIG. 5. Different stability layers of the flow after the generalized Rayleigh circulation criterion: (a) for small values of Ro, two unstable layers (the inner corresponds to rotation-induced perturbations and the outer to centrifugally ariven perturbations) alternate with two stable layers as in the Taylor-Dean system; (b) for large negative Ro, the only potentially unstable layer corresponds to rotation-induced instability and decreases as $|\mathrm{Ro}|$ increases; (c) for positive Ro, the only unstable layer corresponds to centrifugal instability and decreases as Ro increases. 
even in the absence of rotation. Adding rotation will then enhance the effect of the centrifugal force to induce instability. For Ro $<-1.5$, the flow also consists of two layers, with the one close to the inner cylindrical wall $(x=0)$ unstable to rotation-induccd perturbations even in the absence of the curvature (plane Poiseuille channel flow). The centrifugal force enhances the Coriolis force inducing the instability. For intermediate values $R o \in[-1.5,0]$, there are two potentially unstable layers alternating with two stable layers [Fig. $5(a)$. In that case, the competition of the destabilizing mechanisms in the two layers leads to the occurrence of the oscillatory modes. The rotation annihilates the effect of the curvature when the net flux of the equivalent velocity across the gap is zero: $\int_{0}^{1} V_{e} d x=0$, this happens when the rotation ratio $\mathrm{Ro}_{0}=-1$. In the phase diagram $\left(\widetilde{\mathrm{R}}_{\mathrm{o}}, \mathrm{De}_{\boldsymbol{c}}\right)$, the stability branches intersect near the point $\widetilde{R} o=-\delta$. This explains why Matsson and Alfredsson ${ }^{15}$ found, for the gap size $\delta=0.025$, the critical oscillatory modes in the neighborhood of the point $\widetilde{R} 0=-0.028$.

The general linear stability equations (5)-(8) reduce in this case to

$$
\begin{gathered}
{\left[D^{2}-q^{2}-i \operatorname{De}\left(\widetilde{\omega}+p V_{e}\right)\right]\left(D^{2}-q^{2}\right) u} \\
+i p \operatorname{De} D^{2} V_{e} u-2 q^{2} \operatorname{De} V_{e} v=0
\end{gathered}
$$

$$
\left[D^{2}-q^{2}-i \operatorname{De}\left(\widetilde{\omega}+p V_{e}\right)\right] v-\operatorname{De} D V_{e} u=0,
$$

where $\widetilde{\omega}=\omega / \mathrm{De}-p$ Ro. These equations describe centrifugal or rotation-induced instability in the flow whose base flow is described by the equivalent velocity profile $V_{e}$.

The calculation of the eigenfunctions for stationary axisymmetric perturbations $(\omega=0=p)$ shows that for Ro $>-1$, the center of the instability activity belongs to the centrifugally unstable layer and that for Ro $<-1$, it belongs to the rotation unstable layer. Therefore, for $-1 / 2 \delta<$ Ro $<-1.5$, the centrifugal efrects enhance the instability due to the rotation while for $0 \leqslant \operatorname{Ro}<1 / 2 \delta$, the rotation enhances the centrifugal instability.

The analogy with the Taylor-Dean system (flow between two horizontal coaxial rotating cylinders with an external pressure gradient) should be developed. The special case of zero net azimuthal flow studied in Ref. 9 is described in the small gap approximation by equations similar to Eqs. (12) and (13) with the bulk base flow given by $V_{\mathrm{TD}}(x)=3(1+\mu) x^{2}-2(2+\mu) x+1$, where $\mu$ is the rotation ratio of the cylinders. In particular, the two systems become dynamically equivalent for $\mathrm{Ro}=-1$ and $\mu=1$ when the velocity profiles coincide $\left[V_{\mathrm{TD}}(x)=-V_{e}(x)\right]$. The base bulk flow velocity profile in the Taylor-Dean system and the equivalent velocity profile in the rotating curved channel flow have alternating potentially unstable and stable layers in the gap. Interaction between destabilizing mechanisms may lead to oscillatory instability. In spite of this similarity, there are also some differences between these two systems: there is no coupling between the rotation ratio $\mu$ and the curvature factor $\delta$ and the instabilities occurring in the Taylor-Dean system are purely of the centrifugal nature.

\section{Small gap approximation and high rotation regime}

In the small gap approximation and for large values of the rotation number such as $\delta$ Ro $O(1)$, the Rayleigh discriminant becomes

$$
\Phi(x)=2 \delta \operatorname{Ro}\left(D V_{0}+2 \delta \mathrm{Ro}\right) \text {. }
$$

Analysis of this expression indicates that instability will take place in the channel provided $|\mathrm{Ro}|<3 / \delta$. In fact, for large negative $\mathrm{Ro}$, the unstable layer is given by $0<x<x_{0}$ and its extension decreases with increasing Ro until it vanishes at the inner boundary $(x=0)$ as $\mathrm{R} n \rightarrow-3 / \delta$ [Fig. 5(b) ]. For large positive Ro, the unstable layer is given by $x_{0}=1 / 2-\delta \mathrm{Ro} / 6<x<1$ and its extension decreases with increasing Ro until it vanishes at the outer boundary $(x=1)$ as $\mathrm{Ro} \rightarrow 3 / \delta$ [Fig. 5(c) ]. In all these cases, the flow is unstable to axisymmetric perturbations governed by the following equations:

$$
\begin{aligned}
& \left(D^{2}-q^{2}\right)^{2} u-2 q^{2} \operatorname{De} \operatorname{Ro} v=0, \\
& \left(D^{2}-q^{2}\right) v-\operatorname{De}\left(D V_{0}+2 \delta \operatorname{Ro}\right) u=0 .
\end{aligned}
$$

The last system is the same as the one describing rotating plane channel flow ${ }^{11}$ where $D V_{0}=6(1-2 x)$. The critical value $\mathrm{De}_{c}$ increases with $\delta$ Ro, i.e., there is a restabilization effect due mainly to the term $2 \delta$ Ro in Eq. (19). This term expresses the coupling between the curvature and large values of the rotation ratio. The asymptotic behavior of the stability branches in the phase diagram ( $\left.\delta \mathrm{Ro}, \mathrm{De}_{c}\right)$ may be found by using the dynamical similarity of Eqs. (15) and (16) with Taylor-Couette system equations. ${ }^{16}$ In fact, with the correspondence

$$
1-\mu \leftrightarrow 6 /(3-\delta|\mathrm{Ro}|), \quad T \leftrightarrow 4|\mathrm{Ro}|\left(3-\delta \mid \mathrm{Ro}^{\prime}\right) \mathrm{De}^{2}
$$

where $\mu$ is the rotation ratio of cylinders and $T$ is the Taylor number in the Taylor-Couette system, one obtains the following asymptotic relations of the critical parameters for $\delta \mid \operatorname{Ro}[\rightarrow 3:$

$$
\mathrm{De}_{c} \simeq \frac{39.7}{|\mathrm{Ro}|^{1 / 2}(1-\delta|\mathrm{Ro}| / 3)^{5 / 2}}, \quad q_{c} \approx \frac{12.08}{3-\delta|\mathrm{Ro}|}
$$

For $|\mathrm{Ro}|>3 \delta$, the flow has no potentially unstable layer in the gap and no perturbations can develop in the flow. It remains two dimensional in the Taylor-Proudman regime. In fact, except for small values of Ro, the critical wave number increases monotonically with $|\mathrm{Ro}|$ for large values so that the Taylor-Proudman regime corresponds to a flow with vanishing disturbance wavelength.

\section{Wide gap size and rotation regimes}

For wide gap size $\delta>0.01$, the behavior of stability branches for the low rotation regime is similar to that of the small gap approximation. But for higher values Ro, the coupling between rotation and curvature leads to the asymmetry between positive and negative rotation directions (Fig. 3). In that case, phase diagrams $\left(\delta \mathrm{Ro}^{\mathrm{D}} \mathrm{e}_{\mathrm{c}}\right)$ show that the flow is most stable for negative Ro. 


\section{E. Coriolis effects on other hydrodynamic instabilities}

For the circular Couette flow in the rotating frame of reference, for moderate rotation number values, the profile is modified as follows: $V_{e}(x)=V(x)+$ Ro $=1+$ Ro $-(1-\mu) x$, where $\mu$ is the rotation ratio of the cylinders. The generalized Rayleigh criterion and the asymptotic stability equations (15) and (16) indicate that the flow will be unstable for $-1<\operatorname{Ro}<(1-\mu) / 2 \delta$. There is no experimental evidence of this result. A more interesting case is the Taylor-Couette flow subjected to a rotation whose axis is perpendicular to the flow axis. This case provides a rich variety of bifurcations observed in experiments and in theoretical studies. ${ }^{17,18}$

The rotation of the boundary-layer flow on a concave wall enhances the centrifugal instability for moderately positive values of Ro and acts against it for moderately negative values. The boundary-layer flow over a convex plate that is centrifugally stable becomes unstable to rotation-induced perturbations for $-1<$ Ro $<0$ if, while applying the generalized Rayleigh criterion for boundary layers of small thickness, one approximates the boundary-layer flow velocity profile by a piecewise function. ${ }^{25}$

Recently, a lot of numerical simulations have been made for rotating square channel flow ${ }^{26}$ and rotating rectangular channel flow. ${ }^{27}$ The combined effect of the Coriolis acceleration and centrifugal acceleration on flow in ducts has been studied. ${ }^{28}$ All these simulations show that rotation induces longitudinal cells in the flow. Rotation of circular pipe flow about its axis causes destabilization of the laminar HagenPoiseuille flow, ${ }^{29}$ while it suppresses the turbulence and reduces the hydraulic loss. ${ }^{30}$

For thermal convection in fluids with small Prandtl numbers, rotation may induce time-dependent modes at the onset of instabilities. ${ }^{16}$ In binary mixtures, rotation delays the onset of convection and distorts the convective cells. ${ }^{31}$ However, the coupling mechanisms between rotation and thermal convection are different from those occurring in rotating curved channel flows.

\section{F. Oscillatory modes in flows with two control parameters}

In the context of hydrodynamic stability, systems with a transition from the base state to a time-dependent state (Hopf bifurcation) have drawn much attention, because the transition to chaos in those systems is faster than in those with a transition to a stationary state. We list here some of those systems in which competition between several destabilizing mechanisms lead to oscillatory instability: the hydromagnetic Taylor-Couette system, ${ }^{32}$ convection in thermocapillary liquid layers, ${ }^{33}$ thermal convection in an internally heated water layer around its density maximum, ${ }^{34}$ thermal convection in binary fluid mixtures, ${ }^{35}$ thermal convection in two superposed fluid layers, ${ }^{36}$ the Taylor-Dean system described above, and the rotating curved channel flow. The interaction occurs between either an external field (rotation, magnetic field) and a driving mechanism in the flow system or between two driving mechanisms inherent to the flow (e.g., Taylor and Dean instabilities in a Taylor-Dean system). Even if intuitively it is understood that in the vicinity of a codimension-two point (where two stationary neutral curves intersect in the phase diagram), oscillatory modes may occur. Though this has been mathematically described, ${ }^{37}$ the understanding of the physical process that generates the oscillations is still an open problem.

\section{v. CONCLUSION}

The rotating curved channel flow with a finite gap size has been investigated. For small gap size $(\delta<0.01)$, the asymptotic behavior of the stability branches for negative and positive Ro exhibits a reasonable symmetry. For large gap size, the stability branches exhibit asymmetry which grows with the gap size $\delta$. A generalized Rayleigh criterion of stability has been formulated and gives a framework to understand the stability diagram when rotation and curvature effects compete to induce instability.

Curved streamline flows under rotation are often met in technical problems like rotating machines, in geophysical applications, ${ }^{24}$ and in chromatography (field flow fractionation.$^{38}$

\section{ACKNOWLEDGMENTS}

The authors would like to thank M. Martin for many discussions about the applications of these results in chemical systems and N. Stephens for interesting comments on this work.

One of us (I. M.) acknowledges financial support from NATO.

${ }^{1}$ G. I. Taylor, "Stability of a viscous liquid contained between rotating cylinders," Philos. Trans. R. Soc. London Ser. A 223, 289 (1923).

${ }^{2}$ W. R. Dean, "Fluid motion in curved channel," Proc. R. Soc. London Ser. A 121, 402 (1928).

${ }^{3}$ W. H. Reid, "On the stability of viscous flow in a curved channel," Proc. R. Soc. London Ser. A 244, 186 (1958).

${ }^{4}$ R. D. Gibson and A. E. Cook, "The stability of curved channel flow," Q. J. Mech. Appl. Math. 27, 149 (1974).

${ }^{5}$ B. Brewster, P. Grosberg, and A. H. Nissan, "The stability of viscous flow between horizontal concentric cylinders," Proc. R. Soc. London Ser. A 251, 76 (1959).

${ }^{\circ}$ M. D. Kellher, D. L. Flentie, and R. J. MacKee, "An experimental study of the secondary flow in curved rectangular channel," Fluid Fng. (ASME Trans.) 102, 92 (1980)

${ }^{7}$ P. M. Ligrani and R. D. Niver, "Flow visualization of Dean vortices in a curved channel with 40 to 1 aspect ratio," Phys. Fluids 31, 3605 (1988).

${ }^{8}$ I. Mutabazi, J. J. Hegseth, C. D. Andereck, and J. E. Wesfreid, "Pattern formation in the flow between two horizontal coaxial cylinders with a partially filled gap," Phys. Rev. A 38, 4752 (1988).

${ }^{9}$ I. Mutabazi, C. Normand, H. Peerhossani, and J. E. Wesfreid, "Oscillatory modes in the flow between two horizontal corotating cylinders with a partially filled gap," Phys. Rev. A 39, 763 (1989).

${ }^{10} \mathrm{~J}$. E. Hart, "Instability and secondary motion in a rotating channel flow," J. Fluid Mech. 45, 341 (1971).

" D. K. Lezius and J. P. Johnston, "Roll-cell instabilities in rotating laminar and turbulent channel flows," J. Fluid Mech. 77, 153 (1976).

${ }^{12}$ P. H. Alfredsson and H. Persson, "Instabilities in channel flow with system rotation," J. Fluid Mech. 202, 543 (1989).

${ }^{13} \mathrm{~W}$. H. Finlay, "Perturbation expansion and weakly nonlinear analysis for two-dimensional vortices in curved or rotating channels," Phys. Fluids A 1,854 (1989). 
${ }^{14}$ M. C. Potter and M. D. Chawla, "Stability boundary layer flow subject to rotation," Phys. Fluids 14, 2278 (1971).

${ }^{15}$ O. J. F. Matsson and P. H. Alfredsson, "Curvature-and rotation-induced instabilities in channel flow," J. Fluid Mech. 210, 537 (1990):

${ }^{16} \mathrm{~S}$. Chandrasekhar, Hydrodynamic and Hydromagnetic Stability (Oxford U. P., London, 1961).

${ }^{17}$ R. J. Wiener, P. W. Hammer, C. E. Swanson, and R. J. Donnelly, "Stability of Taylor-Couette flow subject to an external Coriolis force," Phys. Rev. Lett. 64, 1115 (1990).

${ }^{18}$ L. Ning, M. Tveitereid, G. Ahlers, and D. S. Cannell, "Taylor-Couette flow subjected to external rotation," Phys. Rev. A 44, 2505 (1991).

${ }^{19}$ L. D. Landau and E. M. Lifshitz, Fluid Mechanics (Pergamon, New York, 1987).

${ }^{20} \mathrm{P}$. H. Roberts, "Experiments on the stability of viscous flow between rotating cylinders. Appendix," Proc. R. Soc. London Ser. A 283, 550 (1965).

${ }^{21}$ W. F. I Langford, R. Tagg, E. J. Kostelich, H. L. Swinney, and M. Golubitsky, "Primary instability and bicriticality in flow between counter-rotating cylinders," Phys. Fluids 31, 776 (1988).

${ }^{22}$ E. M. Sparrow, "On the onset of flow instability in a curved channel of arbitrary height," Z. Angew. Math. Phys. (Kurze Mitteinlugen) 15, 638 (1964).

${ }^{23}$ D. J. Tritton, Physical Fluid Dynamics (Clarendon, Oxford, 1988).

${ }^{24}$ D. J. Tritton and P. A. Davis, "Instabilities in geophysical fluid dynamics," in Hydrodynamic Instabilities and the Transition to Turbulence, Topics in Applied Physics, edited by H. L. Swinney and J. P. Gollub (Springer-Verlag, Berlin, 1981), Vol. 45, p. 229.

${ }^{25}$ P. G. Drazin and W. H. Reid, Hydrodynamic Stability (Cambridge U.P., Cambridge, 1981), Chap. III.

${ }^{26} \mathrm{H}$. S. Kheshgi and L. E. Scriven, "Viscous flow through a rotating square channel," Phys. Fluids 28, 2968 (1985).
${ }^{27} \mathrm{~K}$. Nandakumar, H. Raszillier, and F. Durst, "Flow through rotating rectangular ducts," Phys. Fluids A 3, 770 (1991).

${ }^{28}$ P. Daskopoulos and A. M. Lenhoff, "Flow in curved ducts. Part 2. Rotating ducts," J. Fluid Mech. 217, 575 (1990).

${ }^{29} \mathrm{~J}$. T. Pedley, "On the instability of viscous flow in a rapidly rotating pipe," J. Fluid Mech. 35, 97 (1969).

${ }^{30} \mathrm{G}$. Reich, B. Weigand, and H. Beer, "Fluid flow and heat transfer in an axially rotating pipe-II. Effects of rotation on laminar pipe flow," Int. J. Heat Mass Transfer 32, 551 (1989).

${ }^{31} \mathrm{~K}$. Kumar, "Convective patterns in rotating binary mixtures," Phys. Rev. A 41, 3134 (1990).

${ }^{32}$ T. S. Chang and W. Sartory, "On the onset of instability by oscillatory modes in hydromagnetic Couette flow," Proc. R. Soc. London Ser. A 301, 451 (1967).

${ }^{33}$ M. K. Smith and S. H. Davis, "Instabilities of dynamic thermocapillary liquid layers," J. Fluid Mech. 132, 119 (1983).

${ }^{34} \mathrm{C}$. Normand and A. Azouni, "Penetrative convection in an internally heated layer of water near the maximum density point," Phys. Fluids A 4, 243 (1992).

${ }^{35}$ H. R. Brand, P. C. Hohenberg, and V. Steinberg, "Codimension-2 bifurcation for convection in binary fluid mixtures," Phys. Rev. A 30, 2548 (1984).

${ }^{36}$ S. Rasenat, F. H. Busse, and I. Rehberg, "A theoretical and experimental study of double-layer convection," J. Fluid Mech. 199, 519 (1989).

${ }^{37}$ P. Coullet and E. A. Spiegel, "Amplitude equation for systems with competing instabilities," SIAM J. Appl. Math. 43, 777 (1983).

${ }^{38}$ I. Mutabazi, J. E. Wesfreid, and M. Martin, "Avoidence of hydrodynamic centrifugal instabilities in sedimentation field flow fractionation" in Synopsis of the Second International Symposium on Field Flow Fractionation, Salt Lake City, 1991 (unpublished). 